\title{
Peertechz
}

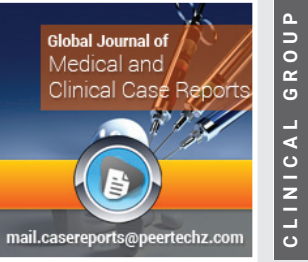

\section{A Case of Mistaken}

\section{Identity: Antiphospholipid Syndrome with Predominant Neuropsychiatric Features}

\author{
Grace E Jackson* and Sedona AZ
}

Received: 31 October, 2020

Accepted: 07 November, 2020

Published: 09 November, 2020

*Corresponding author: Grace E Jackson, Sedona AZ 127 Linda Vista Sedona AZ 86336, USA, Tel: 928554 1022;

Email: grace.e.jackson@att.net, gjsedona@gmail.com Keywords: Antiphospholipid syndrome; Autoimmune disease; Bipolar disorder; Catatonia; Cerebrovascular disease; Dementia; Diagnostic clarification; Schizophrenia

https://www. peertechz.com

\section{Check for updates}

Sedona AZ 127 Linda Vista Sedona AZ 86336, USA

\begin{abstract}
Despite the known association between autoimmune and psychiatric conditions, pertinent evaluations are often overlooked in clinical practice. The author of this report describes the successful identification and treatment of a patient whose myriad neuropsychiatric symptoms were ultimately attributable to antiphospholipid syndrome. The importance of comprehensive and persistent workups is emphasized, particularly in the context of individuals with pre-existing inflammatory diseases and/or cerebrovascular anomalies.
\end{abstract}

\section{Introduction}

The Antiphospholipid Syndrome (APS) is an autoimmune, multi-systemic condition first described by Hughes et al in 1983 [1]. Classically, the diagnosis has required a history of recurrent gestational losses, venous and/or arterial thromboses, and low platelets, combined with the detection of anti-phospholipid antibodies [namely, anti-cardiolipin, lupus anticoagulant, anti-B2 glycoprotein] on 2 or more occasions, at least 12 weeks apart. Although APS can exist as a stand-alone disease, it is not uncommonly detected in conjunction with other medical conditions, such as systemic lupus erythematosus, temporal arteritis, and Raynaud's phenomenon. It has also been associated with infections (including COVID19), medications (i.e., chlorpromazine), and malignancies [2-5].

The involvement of the Central Nervous System in APS has been a common finding. Seizures, headaches, episodes of Transient Global Amnesia (TGA), TIAs, and/or strokes have been attributed to the prothrombotic effects of antiphospholipid antibodies via their interactions with endothelial cells. Additional lines of research have implicated antibody-mediated disruption of brain function via direct binding to neurons and glia, alterations in the permeability of the blood-brain barrier, and/or activation of inflammatory cytokines [3,6].
Non-vascular (non thrombotic) manifestations of APS have increasingly been documented in case reports and observational studies. Here, researchers have found associations between elevated titers of antiphospholipid antibodies and the emergence of dementia, cognitive dysfunction (i.e., poor concentration), mania, depression, movement disorders (chorea, parkinsonism), and psychosis.

Treatments include anti-thrombolytics for patients with histories of arterial and/or venous thrombosis, or immunomodulating medications (such as steroids or antimalarials) for non-vascular presentations. In some cases, the neuropsychiatric features of APS have been found to improve with the early use of these non-psychopharmacologic therapies.

\section{Case presentation}

Ms. X was a 66 year old female whose medical history included hypertension, hyperlipidemia, herpes zoster, joint pain, Giant cell arteritis, Raynaud's phenomenon, and myositis (statin induced). Psychiatric history was notable for ten years of exposure to an antidepressant medication (paroxetine) for symptoms of depression. The patient was a former smoker of cigarettes, abstinent for more than 20 years. Alcohol use consisted of two glasses of wine with evening meals. Home- 
grown cannabis (edibles) had been consumed intermittently over the preceding year as a treatment for joint or muscle pain. A past sensitivity to prednisone -- involving changes in concentration and memory -- had been identified during an episode of temporal arteritis.

In the summer of 2014, Ms. X experienced a critical sequence of medical and treatment-related events:

>> late June: cataract surgery on left eye, followed by post-operative course of prednisone eye drops

>> late July: excessive worrying, increased goal directed activity, insomnia

>> early August: emergency room visit for itchy, burning rash on left side of face

>> anxiety, fatigue, and continuing poor sleep [patient was instructed to discontinue steroid eyedrops]

A follow-up visit to her primary care provider confirmed the presence of slightly red, raised lesions of the left side of the nose and cheek. Titers of varicella zoster virus (VZV) were drawn, and Ms. X was prescribed a one-week course of valcyclovir. After four days, she was instructed to stop the antiviral medication based upon VZV test results (IgG+, IgM negative).

Two weeks later, Ms. X was admitted to the medical floor of the local hospital for the evaluation of cognitive deficits and paranoia. By that time, she was exhibiting severe problems with short term memory (e.g., forgetting what she had cooked $>$ at one point, making three batches of the same soup).

She complained of being unable to think clearly. She would repeat herself over and over. She displayed intermittent periods of decreased mobility and mutism. Diagnostic workup included an analysis of cerebrospinal fluid which was negative for viral or bacterial infections. Carotid ultrasound revealed no evidence of occlusion or stenosis. CT scan of the head was unremarkable. MRI of the brain disclosed minimal white matter lesions. Diffusion weighted images were notable for two foci of increased signal: one at the parieto-occipital junction and one within the left cerebellar hemisphere [Figure 1]. Serial EEGs were obtained. On $8 / 25$, a first tracing displayed mild irregular slowing and sharp activity in the left anterior temporal region. On $8 / 27$, a second study disclosed mild irritability in the left anterior and temporal region with possible epileptogenic potential. Autoimmune tests were not obtained.

Ms. X was transferred to the hospital's behavioral health unit for further treatment of confusion and disorganization. Leading diagnostic considerations were late-onset schizophrenia versus bipolar disorder. Nine days later, she was discharged to home with medications that included oxcarbazepine, paroxetine, lorazepam; and with a pending referral to the author's outpatient psychiatric practice. By late December, a second psychiatric admission was precipitated by worsening catatonia, insomnia, severe cognitive decline, and weight loss ( 23 pounds over three months). While hospitalized,
Ms. X exhibited a cyclic pattern of several hours of excessive energy, followed by protracted periods of social withdrawal, lack of movement, and decreased need for sleep. A Montreal Cognitive Assessment performed on 12/31/14 was scored 5 out of 30 possible points, with deficits in executive functioning, naming, memory, attention, verbal fluency, abstraction, delayed recall, and orientation [Figure 2].
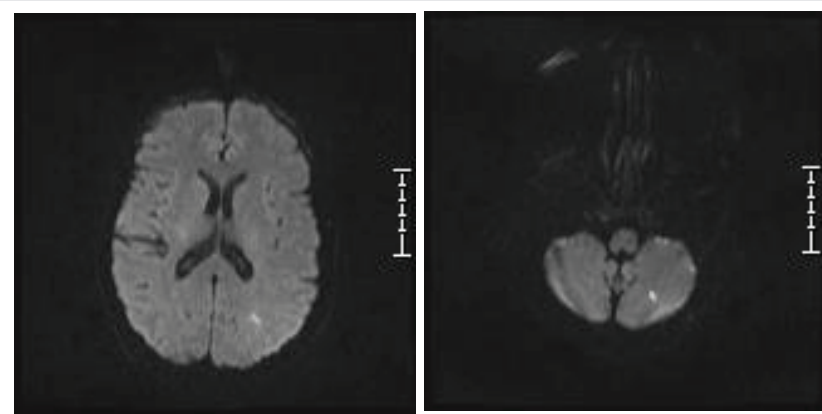

Figure 1: MRI of brain - August 2014.

Interpreted by radiologist as showing non-acute, "likely embolic" foci in left parietooccipital cortex (left) and left cerebellar cortex (right) on axial diffusion weighted images:

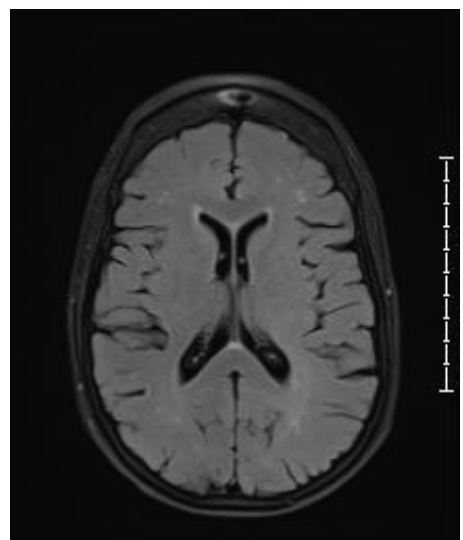

Figure 2: MRI of brain - August 2016

Unremarkable apart from mild, bilateral white matter hyperintensities on axial T2 FLAIR images:

During this second admission, the author directly managed treatments and oversaw an extensive medical workup. MRI of the brain revealed small vessel ischemic changes bilaterally in the frontal and parietal white matter. An MRA of the cerebral vasculature was notable for left vertebral artery atresia. Autoimmune workup revealed +anti ds DNA Ab (61.1), + lupus AC, + ACL antibodies (IgM 33.7), positive P-ANCA and MPO antibodies, and low C4 (6 mg/dL). An autoimmune encephalopathy panel was negative, apart from GAD65 Ab (2.47). Anti TPO and anti-thyroglobulin antibodies were negative. Cancer markers (CA 19-9, CA 125, and AFP) paraneoplastic antibodies (anti-Yo, anti-Ri, anti-Hu), FTA$\mathrm{Ab}, \mathrm{SSA}$ /SSB Ab, RA factor, C-ANCA, ribosomal P Ab, ANA, and CRP were negative. B12 and cortisol levels were within normal limits. Consultations from hospital specialists in neurology and rheumatology were diagnostically inconclusive.

From a clinical perspective, Ms. X's age and chronology of behavioral and cognitive changes suggested a 
neurodegenerative process. A trial of lorazepam was ineffective and triggered disinhibition. Several antipsychotic medications lacked efficacy. Symptoms improved gradually on a regimen of lamotrigine ( $25 \mathrm{mg} \mathrm{qd}$ ) olanzapine (10 $\mathrm{mg}$ qhs), memantine (5 mg qhs), and temazepam (15-30mg qhs prn). The patient was discharged to home with arrangements for physical, occupational, and speech therapies.

Continuing efforts were made by the author to coordinate specialty care for the purpose of diagnostic clarification and treatment optimization.

On $3 / 31 / 16$, lab tests revealed a constellation of autoimmune abnormalities: + anti-cardiolipin antibodies, + beta 2 glycoprotein, elevated alpha 2 macroglobulin, + anti ds DNA $a b$, and positive ANA (1:320, homogeneous pattern). FTA Ab was negative. Sjogren's antibody tests and RF were negative. ESR and CRP were within normal limits. In July, repeat testing revealed low $\mathrm{C}_{4}$, + ANA (1:640), +anti ds DNA Ab, and + anticardiolipin antibodies. Based upon these and previous results, a rheumatologist confirmed the diagnosis of antiphospholipid syndrome and initiated treatment with hydroxycholoroquine.

In August 2016, an MRI of the brain showed mild, scattered white matter hyperintensities [Figure 3]. Magnetic Resonance Angiography revealed an absent ACOM and absent left PCOM. In December 2016, Ms. X experienced a likely episode of transient global amnesia. Repeat lab tests at that time revealed elevated lupus AC, elevated anti-cardiolipin levels, and a positive anti ds DNA Ab. ANA was negative.

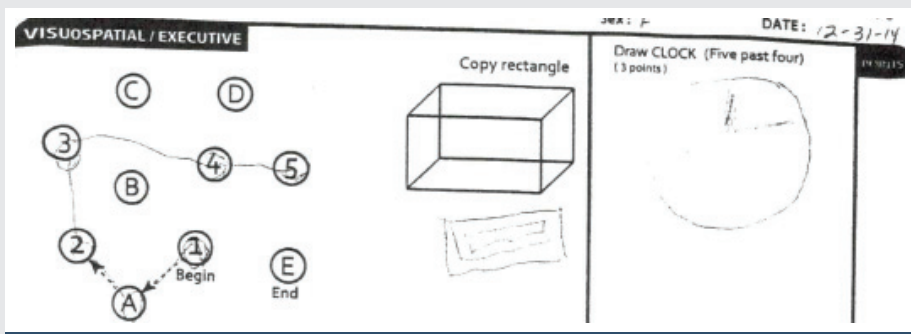

Figure 3: Montreal Cognitive Assessment - December 2014

At the height of the patient's cognitive decline, MOCA was scored 5 out of 30 points. This image shows deficits in visuospatial / executive functions. Ms. $X$ was unable to perform a trail making sequence (far left) and exhibited constructional apraxia when asked to copy a rectangular box (middle). She was unable to depict "five after four" on a clock face:

The patient's behavioral, cognitive, and psychotic features were ultimately stabilized on a regimen of valproic acid (125 $\mathrm{mg}$ bid), clonazepam (0.5 mg bid PRN), doxepin (10 mg qhs prn), and hydroxychloroquine (200 $\mathrm{mg}$ qhs). On 2/8/17, a screening of cognitive functions via the Montreal Cognitive Assessment was scored 28 out of 30 possible points. The patient had resumed volunteer work, regular social interactions with family and friends, and independent functions, such as cooking and driving.

\section{Discussion}

The present case involved a perplexing set of neuropsychiatric features in a 66 year old female. Between
August 2014 and February 2015, an unusual and severe pattern of manic, psychotic, and cognitive symptoms waxed and waned. Initially, it was unclear if Ms. X's catatonia was attributable solely to the effects of steroid eyedrops prescribed after cataract surgery [7-9]. While she had a history of sensitivity to prednisone, exogenous steroids had never before impaired daily functioning. Furthermore, the time course and persistence of symptoms suggested another cause.

The appearance of facial lesions, accompanied by burning and itching in a dermatomal pattern, were consistent with an outbreak of varicella zoster provoked by steroid immunosuppression. The negative IgM antibody result was likely a "false" negative [10]. Importantly, VZV encephalitis was ruled out by a negative spinal fluid exam.

The patient's pattern of alcohol consumption (two glasses of wine nightly) and intermittent cannabis may have contributed to initial mood and/or cognitive changes. However, following the onset of catatonia in the critical summer of the eye surgery, Ms. X adopted complete abstinence from both substances. Even in the aftermath of these behavioral adjustments, her neuropsychiatric difficulties continued.

Serial radiographic studies were obtained. The first MRI of the brain (August 2014) was interpreted as showing non-acute, "likely embolic" foci in the parieto-occipital and cerebellar cortices. These were not visualized on repeat scans. It is possible that these initial abnormalities, in addition to the findings of small vessel ischemia, were reflective of CNS vasculitis associated with APS rather than strokes [11]. MRA findings of anomalies in the cerebral vasculature (left vertebral artery atresia in the first exam; absence of the anterior and posterior communicating arteries in the second) were dismissed by neurology consultants as non-contributory.

Despite the consistent detection of elevated anti ds DNA antibody levels and several occasions of low complement (C4), ANA titers were variable. That finding, combined with a lack of qualifying medical problems (no history of anemia, malar or discoid rash, renal disorder, oral ulcers, photosensitivity, or serositis) precluded the diagnosis of systemic lupus erythematosus [12]. Other autoimmune tests, repeated at intervals, were positive for lupus $\mathrm{AC}$, anti-cardiolipin, and beta 2 glycoprotein antibodies. Consequently, the applicable diagnosis was antiphospholipid syndrome.

Due to the fact that Ms. X lacked a medical history of pregnancy morbidity, arterial or venous thrombosis, thrombocytopenia, migraine headaches, or seizures, her presentation of antiphospholipid syndrome was purely psychiatric at its onset. Perhaps because of this factor, multiple clinicians in various settings, including inpatient hospitalists, failed to consider an autoimmune etiology of symptoms.

\section{Clinical implications}

The resolution of Ms. X's catatonia, manic features, and cognitive deficits depended upon the careful identification of antiphospholipid syndrome, and a synergistic treatment plan consisting of both rheumatologic medication 
(hydroxychloroquine) and psychotropic drugs (valproic acid, clonazepam, doxepin). Had the diagnosis of a primarypsychiatric condition - - such as schizophrenia or bipolar disorder -- been casually accepted, the relevant treatment of the underlying autoimmune disease would have been overlooked. In that scenario, and just as problematically, an aggressive titration of antipsychotic, antiepileptic, or antidepressant medications could have contributed to cerebrovascular or thrombotic events in a patient already at high risk for the same [13-23].

In summary, it is important for clinicians to consider autoimmune pathologies in psychiatric presentations. This is especially true when symptoms are complex, severe, unprecedented, acute in onset, and difficult to stabilize. The evaluation of antiphospholipid syndrome is particularly relevant in patients with evidence of cerebrovascular ischemia, and/or pre-existing inflammatory conditions (such as Giant cell arteritis or Raynaud's phenomenon in the aforementioned case).

\section{Informed consent}

Written informed consent was obtained from the patient for anonymized information to be published in this article.

\section{References}

1. Hughes GRV (2009) Hughes syndrome: a disease of our time. International Journal of Rheumatic Disease 12: 2-4. Link: https://bit.ly/2la5wjw

2. Cervera R (2017) Antiphospholipid syndrome. Thromb Res 151: S43-S47. Link https://bit.ly/3eCe0M8

3. Sanna G, Bertolaccini ML, Cuadrado MJ, Khamashta MA, Hughes GRV (2003) Central nervous system involvement in the antiphospholipid (Hughes) syndrome. Rheumatology 42: 200-213. Link: https://bit.ly/3eLQkW5

4. Raza H, Epstein SA, Pao M, Rosenstein DL (2008) Mania: psychiatric manifestations of the antiphospholipid syndrome. Psychosomatics 49: 438 441. Link: https://bit.ly/2GBhphQ

5. Alhgarthy A, Faqihi F, Balhamar A, Memish ZA, Karakitsos D (2020) Life threatening COVID-19 presenting as stroke with antiphospholipid antibodies and low ADAMTS-13 activity, and the role of therapeutic plasma exchange: a case series. SAGE Open Med Case Rep 8: 2050313X20964089. Link: https://bit.ly/3199h79

6. Gris JC, Nobile B, Bouvier S (2015) Neuropsychiatric presentations of antiphospholipid antibodies. Thromb Res 135: S56-S59. Link: https://bit.ly/354p9SP

7. Kumagai R, Ichimiya Y (2014) Manic episode induced by seroid (fluorometholone) eye drops in an elderly patient. Psychiatry Clin Neurosci 68 : 652-653. Link: https://bit.ly/3n3sZ58

8. Ciriaco M, Ventrice P, Russo G, Scicchitano M, Mazzitello G, et al. (2013) Corticosteroid-related central nervous system side effects. Journal of Pharmacology and Pharmacotherapy 4: S94-S98. Link: https://bit.ly/32lxFLr

9. Dada MU, Oluwole L, Obadeji A, Ajayi OA (2011) Dexamethasone induced psychosis presenting with catatonic features. Afr J Psychiatry (Johannesbg) 14: 316-318. Link: https://bit.ly/38jHe1f

10. Min SW, Kim YS, Nahm FS, Yoo DH, Choi E, et al. (2016) The positive duration of varicella zoster immunoglobulin $\mathrm{M}$ antibody test in herpes zoster. Medicine 95: e4616. Link: https://bit.ly/357BJRe

11. Pomper MG, Miller TJ, Stone JH, Tidmore WC, Hellmann DB (1999) CNS vasculitis in autoimmune disease: MR imaging findings and correlation with angiography. AJNR Am J Neuroradio 20: 75-85. Link: https://bit.ly/3|9FeMJ

12. Anic F, Zuvic-Butorac M, Stimac D, Novak S (2014) New classification criteria for systemic lupus erythematosus correlate with disease activity. Croat Med J 55: 514-519. Link: https://bit.ly/3561W2R

13. Chen PH, Tsai SY, Pan CH, Chang CK, Su SS, et al. (2019) Mood stabilizers and risk of stroke in bipolar disorder. $\mathrm{Br} \mathrm{J}$ Psychiatry 215: 409-414. Link: https://bit.ly/3k8kW4Q

14. Wu CS, Wu KY, Lo YR, Huang YW, Tsai YT, et al. (2018) Psychotropic use and risk of stroke among patients with bipolar disorders: 10-year nationwide population based study. J Affect Disord 226: 77-84. Link: https://bit.ly/3eyevqG

15. Trajkova S, d'Errico A, Soffietti R, Sacerdote C, Ricceri F (2019) Use of antidepressants and risk of incident stroke: a systematic review and metaanalysis. Neuroepidemiology 53: 142-151. Link: https://bit.ly/3ljzikc

16. Schafer W, Princk C, Kollhorst B, Schink T (2019) Antidepressants and the risk of hemorrhagic stroke in the elderly : a nested case control study. Drug Saf 42 1081-1089. Link: https://bit.ly/2U3EL2Q

17. Tully PJ, Alperovitch A, Soumare A, Mazoyer B, Debette S, et al. (2020) Association between cerebral small vessel disease with antidepressant use and depression: 3C Dijon magnetic resonance imaging study. Stroke 51: 402 408. Link: https://bit.ly/3n3tNaa

18. Chen WY, Chen LY, Liu HC, Wu CS, Yang SY, et al. (2017) Antipsychotic medications and stroke in schizophrenia: a case crossover study. PLoS One 12: e0179424. Link: https://bit.ly/3p4UbC2

19. Hsu WT, Esmally-Fard A, Lai CC, Zala D, Lee SH, et al. (2017) Antipsychotics and the risk of cerebrovascular accident: a systematic review and metaanalysis of observational studies. J Am Med Dir Assoc 18: 692-699. Link: https://bit.ly/2GBiRki

20. Letmaier M, Grohmann R, Kren C, Toto S, Bleich S, et al. (2018) Venous thromboembolism during treatment with antipsychotics: results of a drug surveillance program. World J Biol Psychiatry 19: 175-186. Link: https://bit.ly/2U4Emgp

21. Wu CS, Lin CC, Chang CM, Wu KY, Liang HY, et al. (2013) Antipsychotic treatment and the occurrence of venous thromboembolism: a 10year nationwide registry study. J Clin Psychiatry 74: 918-924. Link: https://bit.ly/2U3BoJ6

22. Kunutsor SK, Seidu S, Khunti K (2018) Depression, antidepressant use and risk of venous thromboembolism: systematic review and metaanalysis of published observational evidence. Ann Med 50: 529-537. Link: https://bit.ly/2lhesni

23. Parkin L, Balkwill A, Sweetland S, Reeves GK, Green J, et al (2017) Antidepressants, depression, and venous thromboembolism risk: large prospective study of UK women. J Am Heart Assoc 6: e005316. Link: https://bit.ly/3nOzzZT

Copyright: ( 2020 Jackson GE, et al. This is an open-access article distributed under the terms of the Creative Commons Attribution License, which permits unrestricted use, distribution, and reproduction in any medium, provided the original author and source are credited.

Citation: Jackson GE, Sedona AZ (2020) A Case of Mistaken Identity: Antiphospholipid Syndrome with Predominant Neuropsychiatric Features. Glob J Medical Clin Case Rep 7(2): 109-112. DOI: https://dx.doi.org/10.17352/2455-5282.000111 